\title{
EXPORTAÇÃO DE MACRONUTRIENTES EM CULTIVOS COMERCIAIS DE BAMBU NO TABULEIRO COSTEIRO DO ESTADO DA PARAÍBA ${ }^{1}$
}

\author{
Milton Costa Lima Neto², Egídio Bezerra Neto ${ }^{3}$, Levy Paes Barreto ${ }^{3}$ e José Antonio Aleixo da Silva ${ }^{4}$
}

\begin{abstract}
RESUMO - Amostras de solo e de cavaco de bambu (Bambusa vulgaris) com 11 meses de idade (rebrota) foram coletadas em sítios comerciais localizados no Estado da Paraíba, com o objetivo de avaliar a produção de biomassa de colmos e galhos e o conteúdo e exportação de macronutrientes. O solo foi analisado quanto à fertilidade, e nos cavacos foram efetuadas determinações analíticas dos macronutrientes minerais. A quantidade exportada de cada nutriente foi calculada pela multiplicação do seu teor no cavaco, pela produtividade de cavacos de cada talhão. A produtividade dos cavacos, em toneladas por hectare, foi estimada através da multiplicação da produtividade real, obtida em cada parcela, pelo fator de conversão oriundo da relação entre 1 ha e a área da parcela. O solo da fazenda Mamoaba mostrou teores de Na, K, P, Fe, Zn e Mn superiores aos da fazenda Garapu. O teor de matéria orgânica no solo seguiu esta ordem: Garapu $2>$ Garapu $1>$ Mamoaba. A fazenda Garapu apresentou maior produtividade do que a fazenda Mamoaba, provavelmente devido aos maiores índices pluviométricos. Os cavacos analisados exportaram aproximadamente duas vezes mais $\mathrm{K}$ do que $\mathrm{N}$, sendo $\mathrm{P}$ e S os macronutrientes menos exportados pela cultura. Os teores dos macronutrientes acumulados nos cavacos, bem como a quantidade exportada, seguiram a mesma ordem em todos os sítios estudados: $\mathrm{K}>\mathrm{N}>\mathrm{Ca}>\mathrm{Mg}>\mathrm{P}>\mathrm{S}$. Os programas de adubação da espécie de bambu estudada devem priorizar o fornecimento de nitrogênio, potássio e cálcio.
\end{abstract}

Palavras-chave: Bambusa vulgaris, Nutrientes minerais e Produtividade.

\section{MACRONUTRIENT EXPORTATION IN COMMERCIAL SITES OF BAMBOO AT THE COASTAL TABLELAND OF THE STATE OF PARAÍBA}

\begin{abstract}
Samples of soil and bamboo chips (Bambusa vulgaris) 11 months in age (regrowth) were collected at commercial sites, located in the state of Paraíba (Brazil), aiming to evaluate the stem and branch biomass production, and the content and export of nutrients. The soil was analyzed for fertility and chips had analytical determinations made of mineral nutrients. The export quantity of each nutrient was calculated by multiplying the amount contained in the chip, by the chip productivity of each plot. The chip productivity, in tons per hectare was estimated by multiplying the measured yield of each plot, by the conversion factor derived from the relationship between the area of one hectare and the area of each plot. The soil of the Mamoaba farm showed Na, K, P, Fe, Zn and Mn content higher than the soil from the Garapu farm. The content of organic matter in the soil followed this order: Garapu $2>$ Garapu $1>$ Mamoaba. The Garapu farm had higher productivity than the Mamoaba farm, probably due to the higher rainfall at Garapu farm. The analyzed chips exported roughly twice as much K than N. P and $S$ were the macronutrients less exported by bamboo. The macronutrient contents and exportations followed the same ratio for all sites studied: $K>N>C a>M g>P>S$. The fertilization programs for the studied species of bamboo have to prioritize the supply of nitrogen, potassium and calcium.
\end{abstract}

Keywords: Bambusa vulgaris, Mineral nutrients and Productivity.

\footnotetext{
${ }^{1}$ Recebido em 25.05.2008 e aceito para publicação em 14.10.2009.

${ }^{2}$ Programa de Pós-Graduação em Bioquíma, Universidade Federal do Ceará, UFC, Brasil. E-mail: <miltoncostalima@hotmail.com> .

${ }^{3}$ Universidade Federal Rural de Pernambuco, UFRPE, Brasil. E-mail: <levypaes@yahoo.com.br>.

${ }^{4}$ Fundação de Amparo a Pesquisa do Estado do Amazonas, FAPEAM, Brasil.
} 


\section{INTRODUÇÃO}

Os bambus envolvem várias espécies e gêneros, agrupados na família Poaceae e subfamília Bambusoideae. Encontravam-se distribuídos em cerca de 1.250 espécies e 75 gêneros. Podem crescer como pequenas gramíneas ou chegar à altura de $40 \mathrm{~m}$ (SHAMUGHAVEL e FRANCIS, 2001).

O bambu é amplamente utilizado em todo o mundo. De acordo com Sastry (1998), a cultura movimenta em torno de US\$ 4,5 bilhões por ano. Seus usos são os mais diversos: na construção civil, fabricação de embarcações, móveis, laminados, utensílios domésticos, artesanatos, instrumentos musicais, brinquedos, cestas, alimentação, fitoterápicos e, principalmente, na indústria para a produção de papel de alta resistência (RIBEIRO, 2005).

A espécie Bambusa vulgaris Schrad. ex J.C.Wendl. var. vulgaris, apesar de exótica, originária da China, é a mais utilizada em cultivos comerciais no Brasil. Essa espécie possui colmos grossos, de coloração verde, fibras longas ao longo do colmo e alta produtividade em biomassa nas condições de cultivos no Brasil. Na Região Nordeste são encontradas grandes áreas de cultivos de bambu, a exemplo de Pernambuco, Maranhão e Paraíba, que são grandes produtores da espécie Bambusa vulgaris Schrad. ex J.C.Wendl., destinada à produção de papel de alta resistência. Os Estados do Maranhão e de Pernambuco possuem indústrias de fabricação de papel de bambu, que utilizam toda a produção anual dessa cultura na região.

Mesmo com toda essa crescente demanda, os produtores, pela carência de trabalhos de pesquisa, não possuem conhecimento adequado sobre o manejo e adubação das florestas de bambus. Muitos utilizam as recomendações para outras culturas, como a cana-deaçúcar. A realização de maior número de pesquisas a respeito da adubação e produtividade dos bambus no Brasil é de importância fundamental para o desenvolvimento dessa cultura em nosso país (MENDES, 2004). Conhecer os teores adequados dos nutrientes minerais, bem como o potencial de crescimento e acúmulo de biomassa de bambus para as condições brasileiras, é de suma importância para maior produção com menor custo.

Na Região Nordeste do Brasil, devido ao aumento na procura de bambu como matéria-prima para produção de polpa celulósica, os produtores, além de procurarem aumentar a área de cultivo, têm diminuído o ciclo de corte da cultura. De acordo com Shanmughavel e Francis (2001) e Kleinhenz e Midmore (2001), o manejo ideal para a cultura do bambu, que proporciona maior produtividade, é com o corte e colheita dos colmos com aproximadamente 2,5 anos. Nos cultivos da Região Nordeste, principalmente nos Estados da Paraíba e de Pernambuco, os produtores estão colhendo colmos com apenas 11 meses de cultivo. Essa prática de corte precoce resulta em superexploração das florestas de bambu que, junto com a falta de tratos adequados, acarreta redução paulatina da produtividade nos cultivos comerciais. Em um panorama mundial, grandes países produtores dessa cultura, como Índia, Filipinas e Indonésia, sofrem hoje declínio em suas produções devido à exploração excessiva e precoce dos recursos florestais dos bambuzais. Com a queda na produtividade, os produtores tendem a diminuir cada vez mais o ciclo de cultivo, com o objetivo de tentar atender à demanda industrial. Assim, acaba-se formando um ciclo vicioso e danoso às florestas de bambu. Com a precocidade do corte, cada vez mais diminui a produtividade da cultura (RAMANAYAKE, 2009).

Apesar da vasta utilização do bambu como recurso florestal, a literatura é ainda muito escassa quanto às suas necessidades nutricionais, notadamente nas condições de plantio no Brasil. Este trabalho teve como objetivo relacionar a exportação de macronutrientes essenciais com a produtividade da cultura e o estado de fertilidade do solo, em áreas de cultivos comerciais de Bambusa vulgaris Schrad. ex J.C.Wendl. var. vulgaris, no Estado da Paraíba.

\section{MATERIAL E MÉTODOS}

Foram utilizadas neste trabalho três áreas de cultivos comerciais, em duas fazendas de bambu da espécie Bambusa vulgaris ex J.C.Wendl. var. vulgaris. A fazenda Mamoaba, localizada no Município de Pedras de Fogo (PB), situada aproximadamente a $111 \mathrm{~km}$ ao Norte de Recife (PE), com altitude média de $48 \mathrm{~m}$. Apresenta precipitação pluviométrica anual de $1.300 \mathrm{~mm}$. Sendo a temperatura média anual de $25^{\circ} \mathrm{C}$. A fazenda Garapu, segunda onde foi realizada a pesquisa, está localizada no Município de Alhandra (PB), cerca de 99 km ao Norte da capital pernambucana, com altitude média de 60 $\mathrm{m}$. Apresenta precipitação pluviométrica anual de 1.800 mm e temperatura média anual de $26^{\circ} \mathrm{C}$. 
Nas duas fazendas foram selecionados talhões para a realização das coletas de solo e de cavacos de bambu, formados de colmos e galhos. São eles: Mamoaba - talhão com topografia bastante plana, com 47,0 ha de área; Garapu 1 - talhão com relevo plano, com 34,7 ha de área; e Garapu 2 - talhão com relevo em declive, com 34,0 ha de área. Em cada talhão estudado, foram coletadas cinco amostras compostas de solo, cada uma formada por cinco amostras simples, no perfil de 0 a $20 \mathrm{~cm}$ de profundidade. Utilizou-se uma sonda metálica para a realização das coletas do material. O material foi enviado ao Laboratório de Nutrição Mineral de Plantas da Universidade Federal Rural de Pernambuco, onde se realizaram as análises de fertilidade do solo, de acordo com EMBRAPA (1999).

Cavacos de bambu, formados de colmos e galhos, com idade aproximada de 11 meses de cultivo (plantas de rebrota) foram coletados em cada talhão estudado. Como manejo de rotina da empresa produtora, os bambus eram cortados e deixados no solo por aproximadamente 15 dias para a queda das folhas e uma primeira secagem a céu aberto. Passado esse período, os colmos e galhos são levados a um picador, instalado na sede de cada fazenda, onde são transformados em cavacos para o envio à fábrica produtora de papel.

As amostras de cavaco de bambu foram coletadas diretamente do picador, em intervalos de $5 \mathrm{~min}$, para a obtenção de cinco repetições em cada área de coleta. O material coletado foi levado ao Laboratório de Nutrição Mineral de Plantas da Universidade Federal Rural de Pernambuco.

De posse do material seco e moído, foram realizadas as determinações do resíduo mineral e macronutrientes de acordo com Bezerra Neto e Barreto (2004). As amostras foram submetidas à digestão sulfúrica para determinação de $\mathrm{N}$ e digestão nitricoperclórica para determinação dos demais macronutrientes. O nitrogênio foi determinado pelo método de Kjeldahl, fósforo pelo método colorimétrico do molíbdo-vanadato de amônio, potássio e sódio por fotometria de chama, enxofre por turbidimetria e cálcio e magnésio por espectrofotometria de absorção atômica.

A quantidade exportada de cada nutriente foi calculada através da multiplicação da sua concentração no cavaco, pela produtividade de cavacos de cada talhão analisado, sendo esta última estimada de acordo com Bonilla (1991).
Foram delimitadas parcelas de $15 \mathrm{~m}$ x $15 \mathrm{~m}$, totalizando $225 \mathrm{~m}^{2}$ de área. As touceiras de bambu presentes nessas áreas demarcadas foram cortadas a aproximadamente $5 \mathrm{~cm}$ do nível do solo. Foram separadas as folhas, sendo os colmos e galhos pesados no campo. A produtividade dos cavacos, em toneladas por hectare, foi determinada através da multiplicação da produtividade real, determinada em cada parcela, pelo fator de conversão oriundo da relação entre 1 ha e a área da parcela. Com os dados obtidos, realizaram-se análise descritiva, análise de variância e teste de comparação de médias.

\section{RESULTADOS E DISCUSSÃO}

As três áreas de pesquisa apresentaram solo arenoso, com valores de $\mathrm{pH}$ em torno de 5,5, teores altos de $\mathrm{P}$ e $\mathrm{K}$, baixos teores de $\mathrm{Ca}^{+2} \mathrm{e} \mathrm{Al}^{+3}$, teores adequados de $\mathrm{Mg}^{+2}$, Capacidade de Troca de Cátions (CTC) média e teores baixos de matéria orgânica, de acordo com Camargos (2005) e Malavolta (2006). As amostras de solo coletadas no talhão Mamoaba apresentaram teores de Na, K, P, Fe, Zn e Mn superiores aos das de outros talhões estudados. A concentração de K nas amostras de solo coletadas no talhão Mamoaba foi aproximadamente três vezes maior do que nas amostras coletadas nos outros talhões. O teor de matéria orgânica (\%) no solo seguiu a ordem Garapu $2>$ Garapu 1> Mamoaba entre os talhões analisados (Tabela 1).

Tabela 1 - Análises de fertilidade do solo dos talhões de cultivo de bambu nas fazendas Mamoaba e Garapu.

Table 1 - Soil fertility analysis from bamboo harvest areas at Mamoaba and Garapu farms.

\begin{tabular}{|c|c|c|c|}
\hline \multirow[t]{2}{*}{ CARACTERÍSTICAS } & \multicolumn{3}{|c|}{ ÁREAS DE COLETA } \\
\hline & Mamoaba 1 & Garapu 1 & Garapu 2 \\
\hline $\mathrm{pH}$ & 5,4 & 5,7 & 5,6 \\
\hline $\mathrm{P}\left(\mathrm{mg} \cdot \mathrm{dm}^{-3}\right)$ & 32,3 & 24,2 & 22,1 \\
\hline $\mathrm{K}\left(\mathrm{mg} \cdot \mathrm{dm}^{-3}\right)$ & 94,0 & 32,8 & 31,5 \\
\hline $\mathrm{Na}\left(\mathrm{mg} \cdot \mathrm{dm}^{-3}\right)$ & 15,2 & 10,5 & 9,8 \\
\hline $\mathrm{Zn}\left(\mathrm{mg} \cdot \mathrm{dm}^{-3}\right)$ & 1,8 & 1,2 & 1,4 \\
\hline $\mathrm{Cu}\left(\mathrm{mg} \cdot \mathrm{dm}^{-3}\right)$ & 1,1 & 1,2 & 1,2 \\
\hline Fe $\left(\mathrm{mg} . \mathrm{dm}^{-3}\right)$ & 180,0 & 132,0 & 130 \\
\hline Mn (mg.dm $)$ & 19,4 & 4,2 & 4,6 \\
\hline $\mathrm{H}\left(\mathrm{cmol}_{\mathrm{c}} \cdot \mathrm{dm}^{-3}\right)$ & 2,3 & 2,1 & 2,2 \\
\hline $\mathrm{Al}\left(\mathrm{cmol}_{\mathrm{c}} \cdot \mathrm{dm}^{-3}\right)$ & 0,5 & 0,1 & 0,2 \\
\hline $\mathrm{Ca}\left(\mathrm{cmol}_{\mathrm{c}} \cdot \mathrm{dm}^{-3}\right)$ & 1,1 & 1,6 & 1,5 \\
\hline $\operatorname{Mg}\left(\mathrm{cmol}_{\mathrm{c}} \cdot \mathrm{dm}^{-3}\right)$ & 0,4 & 0,6 & 0,5 \\
\hline $\mathrm{S}\left(\mathrm{cmol}_{\mathrm{c}} \cdot \mathrm{dm}^{-3}\right)$ & 1,9 & 2,3 & 2,3 \\
\hline CTC $\left(\mathrm{cmol}_{\mathrm{c}} \cdot \mathrm{dm}^{-3}\right)$ & 4,8 & 4,5 & 4,5 \\
\hline C (\%) & 0,8 & 1,1 & 1,1 \\
\hline MO (\%) & 1,5 & 1,9 & 2,0 \\
\hline TEXTURA & arenosa & arenosa & arenosa \\
\hline
\end{tabular}

R. Árvore, Viçosa-MG, v.34, n.2, p.251-257, 2010 
O material colhido na fazenda Mamoaba apresentou produtividade de colmos de bambu menor do que os dois talhões da fazenda Garapu (Figura 1). Entre os talhões coletados na fazenda Garapu, o talhão Garapu 1 , que possui topografia plana, apresentou produtividade maior do que o talhão Garapu 2, o qual possui topografia em declive. A topografia da paisagem exerce grande influência sobre alguns fatores climáticos, como radiação e vento, além de maior carreamento dos nutrientes presentes no solo com as chuvas (LARCHER, 2000).

Bonilla (1991) e Mendes (2004), trabalhando com a espécie Bambusa vulgaris Schrad. ex J.C.Wendl. var. vulgaris no Estado de Pernambuco, encontraram maiores valores de produtividade de colmos e galhos, uma vez que as áreas colhidas tinham aproximadamente 15 meses de cultivos, com plantas de rebrota.

Apesar de apresentar produtividade menor, em comparação com os demais talhões estudados, o material colhido no talhão Mamoaba apresentou teor médio do resíduo mineral, aproximadamente duas vezes maior do que os demais materiais colhidos nos outros talhões da fazenda Garapu. Os talhões coletados na fazenda Garapu-Garapu 1 e Garapu 2-apresentaram semelhantes teores do resíduo mineral. Além das particularidades do vegetal, o conteúdo de cinzas foi decorrente da disponibilidade de nutrientes no sítio de cultivo (BARROSO et al., 2005).

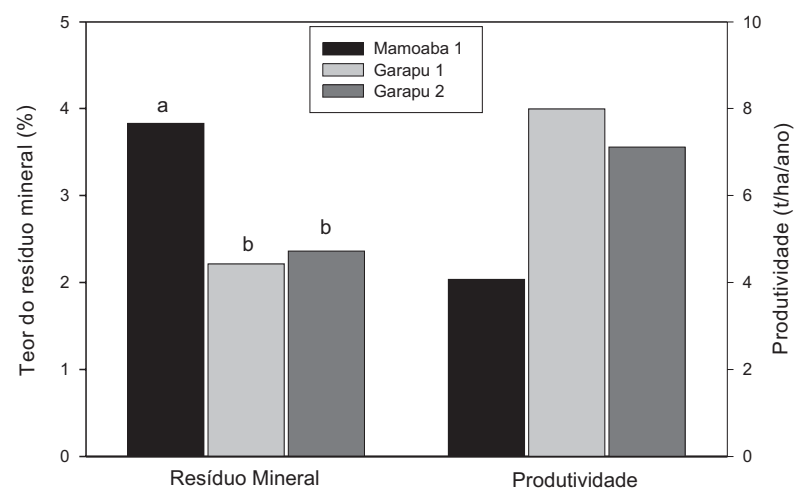

Figura 1 - Teor do resíduo mineral e produtividade de cavacos de bambus colhidos em três áreas de cultivo comercial (safra 2007). Médias com a mesma letra entre as colunas não diferem significativamente $(\mathrm{P}>0,05)$.

Figure 1 - Ash content and productivity of bamboo harvest from commercial tree sites (harvest 2007). Means with the same letter between columns did not differ significantly $(P>0.05)$.
A menor produtividade de cavacos de bambu no talhão Mamoaba pode estar relacionada com as condições climáticas do sítio de cultivo, uma vez que a precipitação pluvial foi menor nesse talhão, em comparação com os demais. No ano em que foram realizadas as coletas dos dados, a precipitação pluvial na fazenda Mamoaba foi $624 \mathrm{~mm}$ a menos do que a precipitação pluvial na fazenda Garapu. Kleinhenz e Midmore (2001) citaram que, na Índia, 61\% de todas as espécies de bambu podem ser encontradas em áreas com 1.500 a 4.000 mm de precipitação pluviométrica por ano. O sucesso no cultivo da espécie Phyllostachyis pubescens Mazel ex J. Houz. em regiões semiáridas na Austrália depende, primariamente, do aporte de mais de 2.000 mm por ano, seja por precipitação, seja por irrigação (KLEINHENZ e MIDMORE, 2001).

Entre os teores dos macronutrientes acumulados nos cavacos de bambu ocorreu diferença significativa $(\mathrm{P}<0,05)$ apenas nos teores de $\mathrm{N}$ entre os materiais coletados nos diferentes sítios de cultivo (Figura 2). Apesar de o solo da fazenda Mamoaba apresentar menor teor de matéria orgânica, o teor de $\mathrm{N}$ nos cavacos foi maior do que nos da fazenda Garapu, possivelmente devido ao sinergismo com o fósforo, cujos teores no solo daquela fazenda foram superiores aos desta. Os teores dos demais macronutrientes não diferiram significativamente entre as áreas de cultivo.

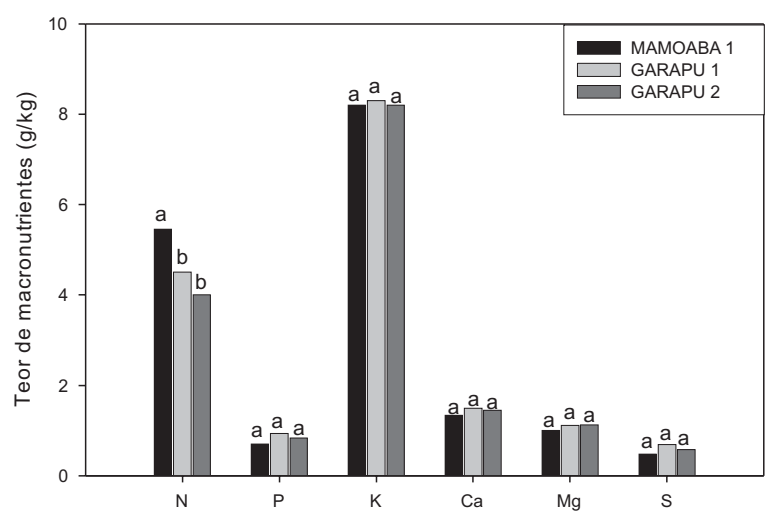

Figura 2 - Teores de nutrientes na matéria seca de cavacos de bambu colhidos em plantios comerciais nas fazendas Mamoaba e Garapu (Safra 2007). Médias seguidas da mesma letra, em cada nutriente, não diferem significativamente $(\mathrm{P}>0,05)$.

Figure 2 - Nutrient contents from dry matter of bamboo chips harvested at commercial sites from Mamoaba and Garapu farms (harvest 2007). Means with the same letters, for each nutrient, did not differ significantly $(P>0.05)$. 
Os cavacos de bambu coletados nas diversas áreas de coleta acumularam os nutrientes analisados na seguinte ordem: $\mathrm{K}>\mathrm{N}>\mathrm{Ca}>\mathrm{Mg}>\mathrm{P}>\mathrm{S}$, proporcionando relação K:N:Ca:Mg:P:S igual a 17,08:11,38:2,79:2,08:1,48:1,00 no talhão Mamoaba, 12,03:6,54:2,17:1,62:1,36:1,00 em Garapu 1 e 14,14:6,90:2,50:1,95:1,45:1,00 no talhão Garapu 2.

Embaye et al. (2005), trabalhando com a espécie Yushania alpina (K.Schum.) W. C. Lin em florestas naturais de bambu na Etiópia, encontraram a seguinte ordem de acúmulo de nutrientes no colmo: $\mathrm{K}>\mathrm{N}>\mathrm{P}>\mathrm{Ca}$, com valores de $\mathrm{K}=9,90 ; \mathrm{N}=4,00 ; \mathrm{P}=0,65$; e Ca = 0,25 g. $\mathrm{kg}^{-1}$. Esses valores estão próximos aos encontrados neste trabalho na espécie Bambusa vulgaris Schrad. ex J.C.Wendl. nas condições de cultivo do Nordeste do Brasil. Já Shanmughavel e Francis (1997) encontraram a ordem $\mathrm{K}>\mathrm{N}>\mathrm{Mg}>\mathrm{Ca}>\mathrm{P}$ em colmos da espécie Bambusa bambos (L.) Voss com um ano de cultivo na Índia. Em Bambusa khasiana Munro, a ordem seguiu a mesma encontrada neste trabalho $\mathrm{K}>\mathrm{N}>\mathrm{Ca}>\mathrm{Mg}>\mathrm{P}$ (RAO e RAMAKRISHNAN, 1989 citados por SHANMUGHAVEL e FRANCIS, 1997).

Quanto à exportação dos macronutrientes, ocorreu diferença significativa entre o talhão Mamoaba e os demais, em todos os macronutrientes. A exportação dos macronutrientes em todas as áreas de cultivos estudadas seguiu a ordem $\mathrm{K}>\mathrm{N}>\mathrm{Ca}>\mathrm{Mg}>\mathrm{P}>\mathrm{S}$, a mesma encontrada nos teores dos nutrientes nas amostras analisadas de cavacos de bambu (Figura 3).

Shanmughavel e Francis (2001) e Kleinhenz e Midmore (2001) encontraram a mesma ordem nos nutrientes exportados, trabalhando com cultivos de bambu na Índia e Austrália, respectivamente. Mendes (2004) comentou que o K é um elemento que pode ser temporariamente armazenado para uso em momentos específicos, o que pode explicar a sua maior exportação. Por se tratar de cultivos jovens em pleno desenvolvimento, a síntese de compostos é alta, garantindo uma demanda por $\mathrm{N}$ e contribuindo, portanto, para uma elevada exportação. O S, entre os nutrientes analisados, foi o que apresentou menor exportação pela cultura. De acordo com os resultados e como citado por Mailly et al. (1997), o bambu acumula aproximadamente duas vezes mais Ca do que $P$, especialmente por sua participação no enrijecimento da parede celular.

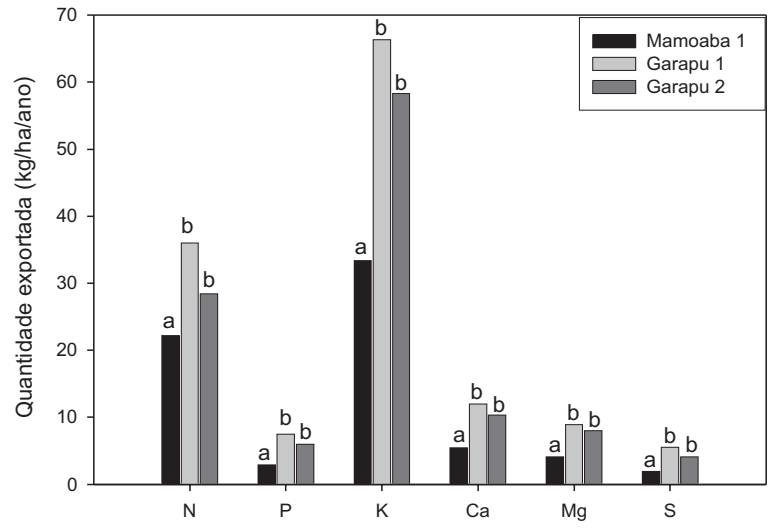

Figura 3-Quantidade de nutrientes exportados pela cultura de bambu em áreas de cultivos comerciais das fazendas Mamoaba e Garapu (Safra 2007). Médias seguidas da mesma letra, em cada nutriente, não diferem significativamente $(\mathrm{P}>0,05)$.

Figure 3 - Exported nutrient contents from bamboo cultures at commercial sites from the Mamoaba and Garapu farm (harvest 2007). Means with the same letter, for each nutrient, did not differ significantly $(P>0.05)$.

O acúmulo e distribuição de nutrientes nos diversos componentes da planta e no solo podem servir de indicadores de diferenças entre os ecossistemas, em especial no que tange à disponibilidade de nutrientes para as plantas (CUNHA et al., 2005). A quantidade de nutrientes exportada por uma cultura está diretamente relacionada com o teor do nutriente no tecido vegetal colhido e com a produtividade da espécie em consideração. Quando ocorre diferença significativa entre as produtividades das áreas de coleta, não necessariamente deve ocorrer diferença significativa na exportação dos nutrientes em kg.ha ${ }^{-1}$. Neste trabalho, o talhão Garapu 1, por possuir maior produtividade de colmos por hectare/ano, apresentou maior taxa de exportação de todos os macronutrientes. Garapu 2 mostrou exportação nutricional de todos os macronutrientes menor do que o talhão Garapu 1 e maior do que o talhão Mamoaba. Este último, por ter conseguido menor produtividade por hectare/ ano, apresentou as menores quantidades de nutrientes exportados, em comparação com os demais talhões analisados.

R. Árvore, Viçosa-MG, v.34, n.2, p.251-257, 2010 


\section{CONCLUSÕES}

De acordo com os resultados deste estudo, chegou-se às seguintes conclusões:

- A quantidade de nutrientes exportada pelo bambu, quando o material colhido era o cavaco, formado por colmos e galhos, obedeceu à seguinte ordem: $\mathrm{K}>\mathrm{N}>\mathrm{Ca}>\mathrm{Mg}>\mathrm{P}>\mathrm{S}$.

- O fator precipitação pluvial exerceu grande influência sobre os valores de produtividade do bambu nos distintos sítios.

- Os programas de adubação da espécie Bambusa vulgaris var. vulgaris devem privilegiar o aporte de nitrogênio, potássio e cálcio.

\section{AGRADECIMENTOS}

Ao CNPq, pela bolsa.

\section{REFERÊNCIAS}

BARROS, N. F.; NEVES, J. C. L.; NOVAIS, R. F. Recomendações de fertilizantes minerais em plantios de eucalipto. In: GONSALVES, J.L.M.; BENEDETTI, V. (Eds.). Nutrição e fertilização florestal. Piracicaba: IPEF, 2000. p.135-165.

BARROSO, D. G. et al. Diagnóstico de deficiência de macronutrientes em mudas de teça. Revista Árvore, v.29, n.5, p.671-679, 2005.

BEZERRA NETO, E.; BARRETO, L. P. Métodos em análises químicas de plantas. Recife: Universidade Federal Rural de Pernambuco, 2004. 148p.

BONILlA, O. H. Análises quantitativas da produção de Bambusa vulgaris Schrader ex Wendland for. vulgaris no estado da Paraíba. 1991. 89f. Dissertação (Mestrado em Botânica) - Universidade Federal Rural de Pernambuco, Recife, 1991.

CALDEIRA, M. V. W. et al. Exportação de nutrientes em função do tipo de exploração e um povoamento de Acacia mearnsii de Wild.

Floresta e Ambiente, v.9, n.1, p.97-104, 2002.

CAMARGOS, S. L. Interpretação de análise de solo. Cuiabá: Universidade Federal do Mato Grosso, 2005. 20p.

R. Árvore, Viçosa-MG, v.34, n.2, p.251-257, 2010
CUNHA, G. M.; GAMA-ROGRIGUES, A. C.; COSTA, G. S. Ciclagem de nutrientes em Eucalypitus grandis W. Hill ex Maiden no Norte Fluminense. Revista Árvore, v.29 n.3, p.353-363, 2005.

EMBAYE, K. et al. Biomass and nutrient distribution in highland bamboo forest in southwest Ethiopia: implications for management. Forest Ecology and Management, v.204, p.159-169, 2005.

\section{EMPRESA BRSILEIRA DE PESQUISA}

AGROPECUÁRIA - EMBRAPA. Manual de análises químicas de solos, plantas e fertilizantes. Brasília: Embrapa Comunicações para transferência de tecnologia, 1999. 370p.

KLEINHENZ, J. ; MIDMORE, D. J. Aspects of bamboo agronomy. Advances in Agronomy, v.74, p.99-149, 2001.

LARCHER, W. Ecofisiologia vegetal. São Carlos: RIMA Artes e Textos, 2000. 531p.

MALAVOLTA, E. Manual de nutrição mineral de plantas. São Paulo: Ceres, 2006. 638p.

MALAVOLTA, E.; VITTI, G. C. ; OLIVEIRA, S. A. Avaliação do estado nutricional das plantas: princípios e aplicações. 2.ed. São Paulo: Potafos, 1997. 319p.

MAILLY, D.; CHRISTANTY, L.; KIMMINS, J. P. Without bamboo, the land dies: nutrient cycling and biogeochemistry of a javanese bamboo talunkebun system. Forest Ecology and Management, v.9, p.155-173, 1997.

MENDES, S. C. Distribuição de biomassa e de nutrientes em plantas comerciais de bambu (Bambusa vulgaris) Schrad, ex Wendl. no nordeste do Brasil. 2004. 45f. Dissertação (Mestrado em Ciências Florestais) - Universidade Federal Rural de Pernambuco, Recife, 2004.

RAMANAYAKE, S. Bamboo grows in stature as nature's green gold. 2009. Disponível em: $<$ http// sundaytimes.lk/090531/Plus/ sundaytimesplus_03.html $>$ acesso em 14 de jun. de 2009.

RIBEIRO, A. S. Carvão de bambu como fonte energética e outras aplicações. Maceió: Instituto do Bambu, 2005.109p. 
SALGADO, A. L. B. Propagação vegetativa de bambu. Instituto Agronômico, v.39, p.17, 1987.

SASTRY, C. B. Bamboo for the $21^{\text {st }}$

century. International Network for Bamboo and Rattan, Beijing, P. R. China, 1998. Disponível em: $<$ http://www.inbar.org.sg/timber.htm> Acesso em: 20 mar. 2006.
SHANMUGHAVEL, P.; FRANCIS, K. Balance and turnover of nutrients in a bamboo plantation (Bambusa bambos) of different ages. Biology Fertility and Soils,. v.25, n.1, p.69-74, 1997.

SHANMUGHAVEL, P.; FRANCIS, K. Physilogy of bamboo. Jodhpur: Scientific Publishers, 2001. 154p. 